\title{
A NOTE ON COMPLEX MONGE-AMPÈRE EQUATION IN STEIN MANIFOLDS*
}

\author{
ALIREZA BAHRAINI ${ }^{\dagger}$
}

\begin{abstract}
We study in this note the Dirichlet problem for complex Monge-Ampère equation in compact Stein manifolds with boundary. As far as we know among the global results for MongeAmpère equations, compact manifolds with boundary have been less discussed.
\end{abstract}

Key words. Monge-Ampère equation, Stein manifold, Continuity method, Pluri-subharmonic functions

AMS subject classifications. 58J32, 35J25

Introduction. We begin this note with a very brief review on some of the aspects of Monge-Ampère equation which have been motivating for the present work. Complex Monge-Ampère equation and its applications have been the subject of extensive studies by several mathematicians since more than 3 decades ago. Thirty years ago S.T.Yau solved complex Monge-Ampère equation on a compact Kähler manifold to prove a conjecture of Calabi:

TheOrem 1. ([15]) Let $X$ be a compact connected Kähler manifold of complex dimension $n$, equipped with a Kähler form $\omega$. If $\mu$ is a smooth volume form satisfying $\mu(X)=\int_{X} \omega^{n}$, then there exists a unique (upto a constant) $\phi \in C^{\infty}(X)$ such that:

$$
(\omega+\sqrt{-1} \partial \bar{\partial} \phi)^{n}=\mu
$$

Since then different variants of the equation for compact or non-compact manifolds have been studied (see [4] [9][13] for example). The solutions of this equation provide us with examples of hyper-Kähler manifolds. In complex dimension $n=2$ the moduli of Hodge structures on K3 surfaces can be characterized locally as well as globally using hyper-Kähler metrics [7] [14]. As a result special Lagrangian submanifolds of $K 3$ surfaces and their properties are much better known and studied. In the category of super-manifolds, an important class of super-symmetric geometries are constructed by the aid of Ricci-flat metrics. This leads to a re-interpretation of special-Lagrangian sub-manifolds in complex dimension 3 and through the works of physicists as bosonic part of super-symmetric objects ([2]).

The problem of studying special Lagrangian representatives for duals of cohomology classes in certain Stein surfaces [2][10] led us to the study of complex MongeAmpère equations in compact Stein manifolds with boundary and to prove the following theorem which seems to be missing in the current literature :

TheOREm 2. Let $X$ be a compact Stein manifold with boundary, $\omega$ a (1,1)-Kähler form on $X, f$ a real smooth function in $X$ and $\phi$ a real smooth function defined only in $\partial X$. Then there is a unique smooth function $u$ on $X$ such that $\omega+\sqrt{-1} \partial \bar{\partial} u>0$ and

\footnotetext{
* Received August 9, 2006; accepted for publication July 17, 2007.

${ }^{\dagger}$ Department of Mathematical Sciences, Sharif University of Technology, P.O. Box 11365-9415, Tehran, Iran (bahraini@sharif.edu).
} 


$$
\begin{aligned}
(\omega+\sqrt{-1} \partial \bar{\partial} u)^{n} & =e^{f} \omega^{n} \\
\left.u\right|_{\partial X} & =\phi .
\end{aligned}
$$

Proof of Theorem 2. In order to prove this theorem we follow the method of Caffarelli, Kohn, Nirenberg and Spruck as in [3] and we find an estimate of the norm $C^{2+\alpha}$ of $u$ for $0<\alpha<1$ :

$$
|u|_{\alpha+2} \leq K
$$

It turns out that for estimates of order 0,1 and $2+\alpha$, some global difficulties arise and the method of Caffarelli et al. needs some modifications.

Estimate of order zero. To show that $|u|_{0}<C$ we need the following lemma:

Lemma 1. Let $u$ and $v$ be two functions in $X$ fulfilling:

$$
\frac{(\omega+\sqrt{-1} \partial \bar{\partial} u)^{n}}{\omega^{n}} \geq \frac{(\omega+\sqrt{-1} \partial \bar{\partial} v)^{n}}{\omega^{n}}
$$

and

$$
u \leq v \text { on } \partial X
$$

then $u \leq v$ in $\bar{X}$.

Proof. In local coordinates we can write

$$
\begin{aligned}
\operatorname{det}\left(g_{i \bar{j}}+u_{i \bar{j}}\right)-\operatorname{det}\left(g_{i \bar{j}}+v_{i \bar{j}}\right) & =\int_{0}^{1} \frac{d}{d t} \operatorname{det}\left(t\left(g_{i \bar{j}}+u_{i \bar{j}}\right)+(1-t)\left(g_{i \bar{j}}+v_{i \bar{j}}\right) d t\right. \\
& =\sum\left(\int_{0}^{1} B^{i \bar{j}}(t) d t\right)(u-v)_{i \bar{j}} \geq 0 .
\end{aligned}
$$

$B^{i \bar{j}}(t)$ are the co-factors of the matrix $\left(t u_{i \bar{j}}+(1-t) v_{i \bar{j}}+g_{i \bar{j}}\right)$ which constitute themeselves a positive definite matrix. So according to the maximum principle $(v-u)$ attains its maximum on $\partial X$ and the desired result follows. $\square$

Now let $\phi_{0}$ be a pluri-subharmonic function in $X$ such that:

$$
\begin{aligned}
\frac{\left(\omega+\sqrt{-1} \partial \bar{\partial} \phi_{0}\right)^{n}}{\omega^{n}} \geq e^{f} & =\frac{(\omega+\sqrt{-1} \partial \bar{\partial} u)^{n}}{\omega^{n}} \\
\left.\phi_{0}\right|_{\partial X} & =\phi .
\end{aligned}
$$

To show the existence of $\phi_{0}$ we take two strictly pluri-subharmonic functions $\phi_{1}$ and $\phi_{2}$ such that $\left.\phi_{1}\right|_{\partial X}=0$ and $\left.\phi_{2}\right|_{\partial X}=\phi$ and we set $\phi_{0}=\lambda \phi_{1}+\phi_{2}$. It's clear that for $\lambda \in \mathbf{R}$ sufficiently large $\phi_{0}$ satisfies the above inequality. Now according to lemma $1, u \geq \phi_{0}$ and we obtain a lower bound for $u$. On the other hand if we calculate the trace of $(\omega+\sqrt{-1} \partial \bar{\partial} u)$ with respect to $\omega$ we find $n+\Delta_{\omega} u \geq 0$. So according to the maximum principle we obtain an upper bound for $u$. 
First order estimates. We would like to show the existence of a bound for norm 1:

$$
|u|_{1}<C
$$

Let $F$ be the application defined by

$$
F(u)=\log \frac{(\omega+\sqrt{-1} \partial \bar{\partial} u)^{n}}{\omega^{n}}-f .
$$

We take a unit tangent vector $\xi$ in a given point of $X$. Let $D$ be a vector field extending $\xi$ which can be described in an open dense holomorphic coordinate subset $U$ of $X$ as a vector field with constant coefficients. Such a field exists: it suffices to imbed $X$ properly in some $\mathbb{C}^{N}$ and then to project over a generic vector sub-space of dimension $n$. Let $h$ be a potential of the metric $\omega$ in some local coordinates near a point $x \in U$. We have:

$$
F(u)=\log \operatorname{det}\left((h+u)_{i \bar{j}}\right)-\log \operatorname{det}\left(h_{i \bar{j}}\right)-f .
$$

Set $k=h+u$ and suppose that $F(u)=0$. It can be easily seen that $F^{i \bar{j}}=\frac{\partial F}{\partial u_{1 \bar{j}}}=$ $\left(k^{i \bar{j}}\right)$ the inverse of the matrix $\left(k_{i \bar{j}}\right)$ and the linearisation of the operator $F$ at $u$ is written as follows:

$$
(\tilde{L}) v, \quad \tilde{L}=k^{i \bar{j}} \partial_{i \bar{j}}
$$

We have $D F=0$ and since $D$ is described almost everywhere in local coordinates by a vector field with constant coefficients, a.e. we get:

$$
\tilde{L}(D k)=D H
$$

where $H=f+\log \operatorname{det}\left(h_{i \bar{j}}\right)$. On the other hand we know that $\operatorname{det}\left(k^{i \bar{j}}\right)=e^{H}$, so

$$
\frac{1}{n} \sum k^{i \bar{i}} \geq e^{H / n}
$$

and for some constant $B_{x}$ :

$$
|D H| \leq B_{x} e^{H / n} \text { near } x .
$$

In order to establish the inequality:

$$
\max _{\bar{X}}|D u| \leq \max _{\partial X}|D u|+C
$$

let $p$ be a strictly pluri-subharmonic function in $X$ and consider locally defined functions $w_{0}^{ \pm}= \pm D k+e^{\lambda p}$ near $x$. If $\eta$ denotes the least eigenvalue of $\left(p_{i \bar{j}}\right)$ we obtain

$$
\begin{aligned}
\tilde{L} w_{0}^{ \pm} & = \pm \tilde{L} D k+\tilde{L} e^{\lambda p}= \pm D H+k^{i \bar{j}}\left(e^{\lambda p}\right)_{i \bar{j}} \\
& = \pm D H+k^{i \bar{j}}\left(\lambda p_{i \bar{j}}+\lambda^{2} p_{i} p_{\bar{j}}\right) e^{\lambda p} \geq-B e^{H / n}+\left(\lambda \eta \sum k^{i \bar{\imath}}\right) e^{\lambda p} .
\end{aligned}
$$

Hence: 


$$
\tilde{L}( \pm D h)= \pm k^{i \bar{j}} D h_{i \bar{j}} \geq-\gamma \sum k^{i \bar{i}}
$$

where $\gamma$ is a constant which depends only on the metric $\omega$. Furthermore for globally defined functions $w_{1}^{ \pm}= \pm D u+e^{\lambda p}$ in $X$, we have $w_{1}^{ \pm}=w_{0}^{ \pm} \mp D h$, thus for all $\mathrm{x}$ in $U$, in a neighborhood of $x$ one gets:

$$
\tilde{L} w_{1}^{ \pm} \geq-B_{x} e^{H / n}+\sum k^{i \bar{i}}\left(\lambda \eta e^{\lambda p}-\gamma\right) .
$$

The compactness of $X$ allows us to find a finite number of points $x$ in $U$ and some neighborhoods of these points covering $U$ on which the metric has local potentials. By using the inequality (5) it follows that for $\lambda$ sufficiently large

$$
\tilde{L} w_{1} \geq 0
$$

and the inequality (6) follows with the aid of the maximum principle.

To complete the demonstration of (3) we should find upper bounds for $|D u|$ on $\partial X$. Let $\phi_{0}$ be a pluri-subharmonic function in $X$ s.t.

$$
\begin{aligned}
\frac{\left(\omega+\sqrt{-1} \partial \bar{\partial} \phi_{0}\right)^{n}}{\omega^{n}} \geq e^{f} & =\frac{(\omega+\sqrt{-1} \partial \bar{\partial} u)^{n}}{\omega^{n}} \\
\left.\phi_{0}\right|_{\partial X} & =\phi
\end{aligned}
$$

and let $\psi$ denote a solution of the equation $\Delta_{\omega} \psi=-n,\left.\psi\right|_{\partial X}=\phi$ then :

$$
\phi_{0} \leq u \leq \psi
$$

and we obtain

$$
|\nabla u(z)| \leq \max \left\{\left|\nabla \phi_{0}(z)\right|,|\nabla \psi(z)|\right\}, z \in \partial X .
$$

Estimates of second order derivatives. Following [3] it is sufficient to show:

Lemma 2. There exists a constant $C>0$ such that:

$$
\max _{\bar{X}}\left|\nabla^{2} u\right| \leq \max _{\partial X}\left|\nabla^{2} u\right|+C .
$$

Proof: Let $D$ be a vector field which can be described almost everywhere by constant coefficients in appropriate coordinate systems. Using the concavity of $F$ as a function of $k_{i \bar{j}}$ locally we get:

$$
\begin{gathered}
k^{i \bar{j}}\left(D^{2} k\right)_{i \bar{j}} \geq D^{2} H \\
\tilde{L} D^{2} k \geq-C H^{-1 / n} .
\end{gathered}
$$

Consequently as we did in the estimations for the first order derivatives, for $\lambda$ sufficiently large we can prove the following inequality:

$$
\tilde{L}\left(D^{2} k+e^{\lambda p}\right) \geq 0
$$


and then use the maximum principle to complete the proof of the lemma.

On the other hand we have

Lemma 3. ([2]) There exists a constant $C$ such that

$$
\max _{\partial X}\left|\nabla^{2} u\right| \leq C
$$

This gives the required bound on second order derivatives.

Estimates of order $(2+\alpha)$. The argument contains the following steps:

Lemma 4. ([3],[4]) For all $X^{\prime} \subset \bar{X}^{\prime} \subset \bar{X} \backslash \partial X$ there exists $K\left(X^{\prime}\right)$ such that:

$$
|u|_{2+\alpha} \leq K\left(X^{\prime}\right) .
$$

Lemma 5. ([2]) There exists a constant $K$ such that for all $x, y \in \partial X$ :

$$
\left|u_{i j}(x)-u_{i j}(y)\right| \leq \frac{K}{1+|\log | x-y||} \text { for } x, y \in \partial X
$$

Using this lemma we prove that:

Lemma 6. There exists a constant $K$ such that for all $x \in \partial X, y \in \bar{X}$ we have:

$$
\left|u_{i j}(x)-u_{i j}(y)\right| \leq \frac{K}{1+|\log | x-y||}
$$

Proof. The idea is again the maximum principle, this time by constructing two functions: one denoted by $w$, and defined in (11), which contains second order derivatives, and the other $v$, defined in (12) and fulfilling $\tilde{L} h<\tilde{L} v$. By (7) they may be so chosen that on $\partial X$ we have $w \leq v$. In this way we obtain a control for $w$ leading to the inequality (8).

Let $\left\{U_{\alpha}\right\}$ be a covering of $X$ such that each $U_{\alpha}$ is biholomorphic with an open set in $\mathbb{C}^{n}$, and let $\left\{f_{\alpha}\right\}$ be an associated partition of unity. According to I.Motzkin and W.Wasow [11] in each $U_{\alpha}$ there exist vector fields of length 1 with constant coefficients $\xi_{\alpha}^{1}, \ldots, \xi_{\alpha}^{N}$ and constants $c_{1}, \ldots, c_{N}$ such that the linear approximation $\tilde{L}_{\alpha}$ of $F$ can be written as:

$$
\tilde{L}_{\alpha}=\sum_{1}^{N} b_{i}^{\alpha}(x) \partial_{\xi_{\alpha}^{i}}^{2} \text { with } c_{i} \leq b_{i}^{\alpha} \leq c_{i}^{-1} .
$$

Further one can suppose that the operators $\partial_{\xi_{\alpha}^{i}}$ contain all the operators $\partial / \partial x_{i}$ as well as $1 / \sqrt{2}\left(\left(\partial / \partial x_{i}\right) \pm\left(\partial / \partial x_{j}\right)\right)$ for $i \neq j$ in the coordinates of $U_{\alpha}$. Then we calculate $\tilde{L}_{\alpha}\left(\partial_{\xi_{\alpha}}\right)^{2} k^{\alpha}$, for a unit vector $\xi_{\alpha}=\left(\xi_{\alpha 1}, \ldots, \xi_{\alpha n}\right)$, by applying $\partial_{\xi_{\alpha}}^{2}$ on both sides of the equation (4). In this way, with the previous notations can write:

$$
\tilde{L}\left(\partial_{\xi_{\alpha}}\right)^{2} k^{\alpha}+F^{i j, p l} \partial_{\xi_{\alpha}} k_{i j}^{\alpha} \partial_{\xi_{\alpha}} k_{p l}^{\alpha}+\partial_{\xi_{\alpha}} H=0 .
$$


Hence by concavity of $F$ and using the inequality $|u|_{2}<C$ we can find some constants $c_{j p}$ such that

$$
\tilde{L}\left(\partial_{\xi_{\alpha}}\right)^{2} k^{\alpha} \geq-C-\sum c_{j p} \partial_{\xi_{\alpha}} k_{j p}^{\alpha}
$$

Let $\epsilon$ be a positive number such that :

$$
\epsilon\left|\nabla^{2} k^{\alpha}\right| \leq \frac{1}{4}
$$

where $\left|\nabla^{2} k^{\alpha}\right|$ represents the norm of the hessian matrix $\left(k_{i j}^{\alpha}\right)$. We have

$$
\tilde{L}\left(\partial_{\xi_{\alpha}}^{2} k^{\alpha}+\epsilon\left(\partial_{\xi_{\alpha}}^{2} k^{\alpha}\right)^{2}\right) \geq-C-C \sum_{i j p}\left|k_{i j p}^{\alpha}\right|+2 \epsilon c_{0} \sum_{i}\left|\partial_{\xi_{\alpha}}^{2} k_{i}^{\alpha}\right|^{2}
$$

thus if we define

$$
h=\sum_{\alpha} \sum_{1}^{N} f_{\alpha}\left(\partial_{\xi_{\alpha}^{i}}^{2} k^{\alpha}+\epsilon\left(\partial_{\xi_{\alpha}^{i}}^{2} k^{\alpha}\right)^{2}\right)
$$

we get the inequality:

$$
\tilde{L} h \geq-C-C \sum_{\alpha} \sum_{i j p}\left|k_{i j p}^{\alpha}\right|+2 \epsilon c_{0} \sum_{\alpha} \sum_{i=1}^{n} \sum_{j=1}^{N}\left|\partial_{\xi_{\alpha}^{j}}^{2} k_{i}^{\alpha}\right|^{2}
$$

which can be deduced from the inequality (9) and from a second order estimation on $k$.

Now since:

$$
\sum_{i} \sum_{j}\left|\partial_{\xi_{\alpha}^{j}}^{2} k_{i}^{\alpha}\right|^{2} \geq c_{1} \sum\left|k_{i j p}^{\alpha}\right|^{2} \text { for } c_{1} \text { positive }
$$

one finds,

$$
\tilde{L} h \geq c_{0} \epsilon \sum\left|k_{i j p}^{\alpha}\right|^{2}-\frac{C}{\epsilon} .
$$

Thus if we define $w^{i}$ by

$$
w^{i}=\sum_{\alpha} f_{\alpha} \partial_{\xi_{\alpha}^{i}}^{2} k^{\alpha}+\epsilon h
$$

for $i=1, \ldots, N$ we obtain

$$
\tilde{L} w^{i} \geq-\frac{C}{\epsilon^{2}} .
$$

Now set $w=w^{i}$. Let $y_{0} \in \partial X$ be fixed and suppose that $X$ is imbedded in $\mathbb{C}^{r}$ such that $y_{0}$ coincides with 0 . Let $\delta=|y|^{1 / 3}$ for fixed $y$ and $g$ be a smooth function in $\bar{X}$ vanishing on $\partial X$ and satisfying $\tilde{L} g \leq-1$ (so $g>0$ in $X$ ). Define:

$$
v(x)=w(0)+\frac{M}{|\log | \delta||}+M \frac{|x|^{2}}{\delta^{2}}+\frac{A g}{\delta^{2}},
$$


where $|$.$| is the norm of \mathbb{C}^{r}$. We have,

$$
\tilde{L} v \leq \frac{C M}{\delta^{2}}-\frac{A g}{\delta^{2}}
$$

and so for $A=C M+C / \epsilon^{2}$,

$$
\tilde{L} v \leq-\frac{C}{\epsilon^{2}}<\tilde{L} w
$$

according to (7) for $x \in \partial X,|x|<\delta$,

$$
|w(x)-w(0)| \leq \frac{C}{|\log | \delta||}
$$

hence for $M$ sufficiently large we get $w \leq v$ on $\partial X$, and the maximum principle yields:

$$
w \leq v \text { in } X .
$$

In particular,

$$
w(y)-w(0) \leq \frac{3 M}{|\log | y||}+M|y|^{4 / 3}+C A|y|^{1 / 3} \leq \frac{C}{|\log | y||} .
$$

Here we use the fact that $g$ is a $C^{\infty}$ function vanishing on the boundary to estimate the last term.

Thus if we choose the partition of unity in such a way that in a neighborhood of $0 \in \partial U_{\alpha} \cap \partial X, f_{\alpha}=1$ then for $j=1, \ldots, N$ we get :

$$
\begin{aligned}
& \partial_{\xi_{\alpha}^{j}}^{2} k(y)-\partial_{\xi_{\alpha}^{j}}^{2} k(0)+\epsilon \sum_{j=1}^{N}\left(\partial_{\xi_{\alpha}^{j}}^{2} k(y)-\partial_{\xi_{\alpha}^{j}}^{2} k(0)\right)\left(1+\epsilon \partial_{\xi_{\alpha}^{j}}^{2} k(y)+\epsilon \partial_{\xi_{\alpha}^{j}}^{2} k(0)\right) \\
\leq & \frac{C}{|\log | y||}
\end{aligned}
$$

After multiplying the relation (13) by $1+\epsilon \partial_{\xi_{\alpha}^{i}}^{2} k(y)+\epsilon \partial_{\xi_{\alpha}^{i}}^{2} k(0)$ and summing over $i$ we find:

$$
h(y)-h(0) \leq \frac{C}{|\log | y||}
$$

but according to our choice of the partition of unity we know that in a neighborhood of 0 in $U_{\alpha}$

$$
h=\sum_{j=1}^{N}\left(\partial_{\xi_{\alpha}^{j}}^{2} k^{\alpha}+\epsilon\left(\partial_{\xi_{\alpha}^{j}}^{2} k^{\alpha}\right)^{2}\right) .
$$

In order to obtain an inequality in the opposite direction we use the concavity of $F$ as a function of $D^{2} k^{\alpha}$ :

$$
\begin{aligned}
F\left(x, D^{2} k^{\alpha}(x)\right)+F^{i j}\left(x, D^{2} k^{\alpha}(x)\right)\left(k_{i j}^{\alpha}(y)-k_{i j}^{\alpha}(x)\right) & \geq F\left(x, D^{2} k^{\alpha}(y)\right) \\
& \geq F\left(y, D^{2} k^{\alpha}(y)\right)-C|x-y| .
\end{aligned}
$$


By using the representation (9) we can rewrite the last inequality as:

$$
\sum_{i=1}^{N} b_{i}^{\alpha}(x)\left(\partial_{\xi_{\alpha}^{i}} k^{\alpha}(y)-\partial_{\xi_{\alpha}^{i}} k^{\alpha}(x)\right) \geq-C_{\alpha}|x-y| .
$$

Setting $x=0$, for $p \leq N$ and by using (13) we find :

$$
\begin{aligned}
b_{p}^{\alpha}(0)\left(\partial_{\xi_{\alpha}^{p}} k^{\alpha}(y)-\partial_{\xi_{\alpha}^{p}} k^{\alpha}(0)\right) \geq & -\sum_{i \neq p} b_{i}^{\alpha}(0)\left(\partial_{\xi_{\alpha}^{i}} k^{\alpha}(y)-\partial_{\xi_{\alpha}^{i}} k^{\alpha}(0)\right)-C|y| \\
= & -\sum_{i \neq p} b_{i}^{\alpha}(0)\left(w^{i}(y)-w^{i}(0)\right. \\
& +\epsilon(h(y)-h(0)) \sum_{i \neq p} b_{i}^{\alpha}(0)-C|y| \\
\geq & \epsilon \sum_{i \neq k} b_{i}^{\alpha}(0) \cdot(h(y)-h(0))-\frac{C}{|\log | y||}
\end{aligned}
$$

from which we get

$$
h(y)-h(0) \geq-\frac{C}{|\log | y||}
$$

Thus we have shown:

$$
|h(y)-h(0)| \leq \frac{C}{|\log | y||}
$$

and therefore:

$$
\left|\partial_{\xi_{\alpha}^{p}} k^{\alpha}(y)-\partial_{\xi_{\alpha}^{p}} k^{\alpha}(0)\right| \leq \frac{C}{|\log | y||}
$$

Since the $\partial_{\xi_{\alpha}^{i}}$ contain all $\partial_{x_{i}}$ and $1 / \sqrt{2}\left(\partial_{x_{i}} \pm \partial_{x_{j}}\right)$ for $i \neq j$ the desired inequality (8) is implied.

The proof of theorem 1 can now be completed by:

Lemma 7. ([2]) If the inequality (8) holds then for a positive number $\alpha<1$ we have:

$$
\left|u_{i j}(x)-u_{i j}(y)\right| \leq K|x-y|^{\alpha}
$$

As a result we obtain:

Corrolary 1. Let $X$ be a Stein manifold with boundary s.t. $K_{X} \cong O_{X}$ then in each class of metric $[\omega] \in A^{1,1}(X) \cap H^{2}(X, \mathbb{R})$ and for every function $\phi \in C^{\infty}(\partial X)$ there exists a unique Ricci-flat metric $\omega^{\prime}$ in the same class as $[\omega]$ such that $\omega^{\prime}=$ $\omega+\sqrt{-1} \partial \bar{\partial} u$ with $\left.u\right|_{\partial X}=\phi$ 
Acknowledgement. I would like to thank Professor Daniel Bennequin for several helpful discussions. I would also thank the research council of Sharif University of Technology for support.

\section{REFERENCES}

[1] T. Aubin, Nonlinear analysis on manifolds, Monge-Ampère equations. Grundlehren der Mathematischen Wissenschaften, 252, Springer-Verlage, New York-Berlin, 1982.

[2] A. Bahraini, Super-symétrie et géométrie complexe, 2004, Ph.D Thesis in University Paris 7 Denis Diderot.

[3] L. Caffarelli, L. Nirenberg and J. Spruck, The Dirichlet problem for nonlinear secondorder elliptic equations II. Complex Monge-Ampère, and uniformly elliptic equations, Comm. Pure App. Math., 38 (1985), pp. 209-252.

[4] P. Eyssidieux, V. Guedj ANd A. Zeriahi, Singular Kähler-Einstein metrics, math.AG/0603431.

[5] L. C. Evans, Classical solutions of fully nonlinear, convex, second order elliptic eqations, Comm. Pure Appl. Math., 35 (1982), pp. 333-363.

[6] D. Gilbarg and N. S. Trudinger, Elliptic Partial Differential Equations of Second Order, Springer-Verlag, Berlin-Heidelberg-New York, 2d ed., 1984.

[7] D. Huybrechts, Compact Hyper-Kähler manifolds: basic results, Invent. Math., 135 (1999), pp. 63-113.

[8] L. HöRMANDER, The analysis of linear partial differential operators III pseudodifferential operators, Grundlehren der Mathematischen Wissenschaften 274 Springer-Verlag, Berlin, 1983.

[9] S. KolodzieJ, The complex Monge-Ampère equation, Acta Math., 180 (1998),pp. 69-117.

[10] T. T. LÊ, Ph.D Thesis, 2002 in University Paris 7 Denis Diderot.

[11] I. Motzkin AND W. WASOW, On the approximation of linear elliptic differential equations by difference equations with positive coefficients, J. Math. and Phys., 31 (1952), pp. 253-259.

[12] G. Tian, Canonical metrics in Kähler geometry, Lectures in Mathematics, ETH Zürich. Birkhäuser Verlag, Basel (2000).

[13] G. TIAn And S.-T. YAU, Complete Kähler manifolds with zero Ricci curvature, Invent. Math, 106 (1991), pp. 27-60.

[14] A. N. Todorov, The Weil-Petersson geometry of the moduli space of $S U(n \geq 3)$, (Calabi-Yau) manifolds, Comm. Math. Phys, 126 (1989), pp. 325-346.

[15] S.-T. YAU, On the Ricci curvature of a compact Kähler manifold and the complex MongeAmpère equation I, Com. Pure and Appl. Math., 31 (1978), pp. 339-411. 
A. BAHRAINI 